\title{
Influence of High Pressure on the Local Order and Dynamical Properties of the Selected Azole Antifungals
}

Dawid Heczko ${ }^{1 *}$, Karolina Jurkiewicz, ${ }^{2,3}$ Joanna Grelska ${ }^{2,3}$, Kamil Kamiński ${ }^{2,3}$, Marian Paluch $^{2,3}$, Ewa Kamińska ${ }^{*}$

${ }^{I}$ Department of Pharmacognosy and Phytochemistry, Faculty of Pharmaceutical Sciences in Sosnowiec, Medical University of Silesia in Katowice, Jagiellońska 4, 41-200 Sosnowiec, Poland

${ }^{2}$ Institute of Physics, University of Silesia in Katowice, 75 Putku Piechoty 1, 41-500 Chorzów, Poland

${ }^{3}$ Silesian Center for Education and Interdisciplinary Research, 75 Putku Piechoty 1A, 41-500 Chorzów, Poland

\section{SUPPORTING INFORMATION}

a)
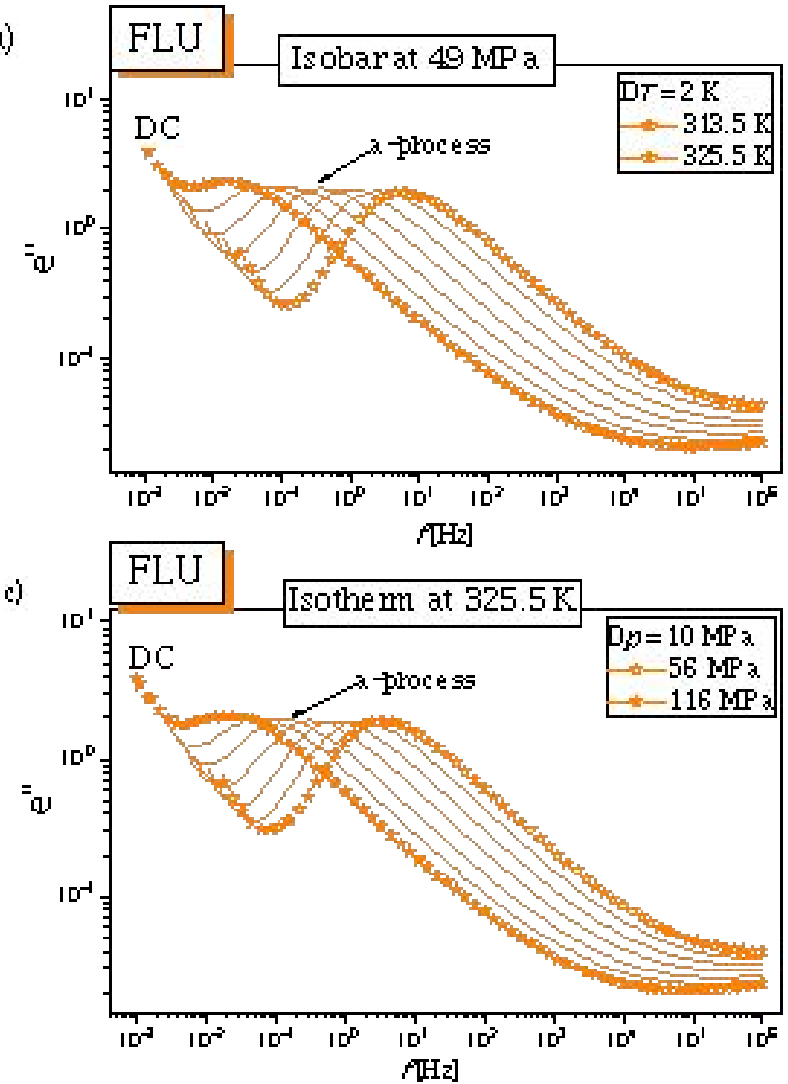

b)

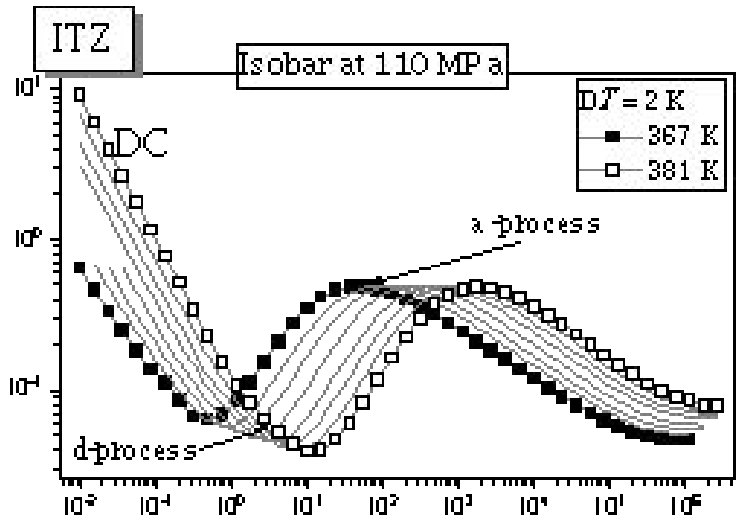

d)

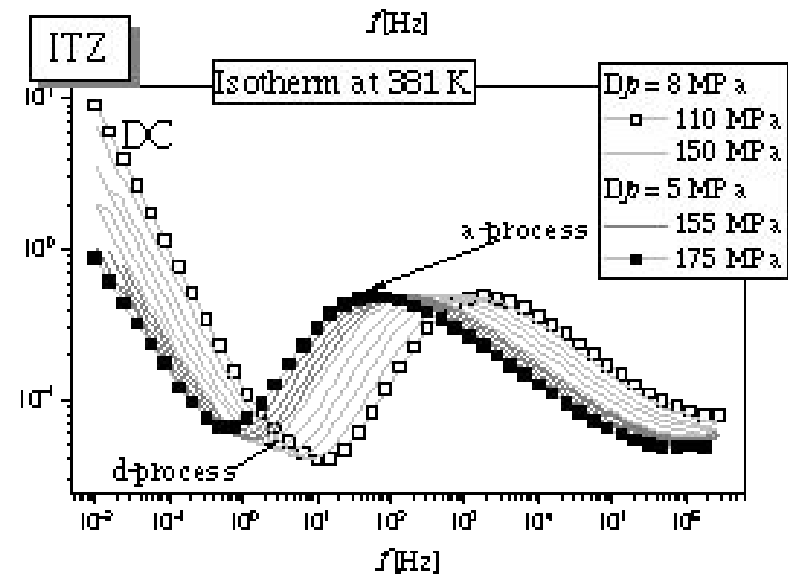

Figure S1. Representative dielectric loss spectra measured for FLU and ITZ at constant pressure and indicated temperatures (panels a and b), and at constant temperature and indicated pressures (panels $\mathrm{c}$ and d). 
a)

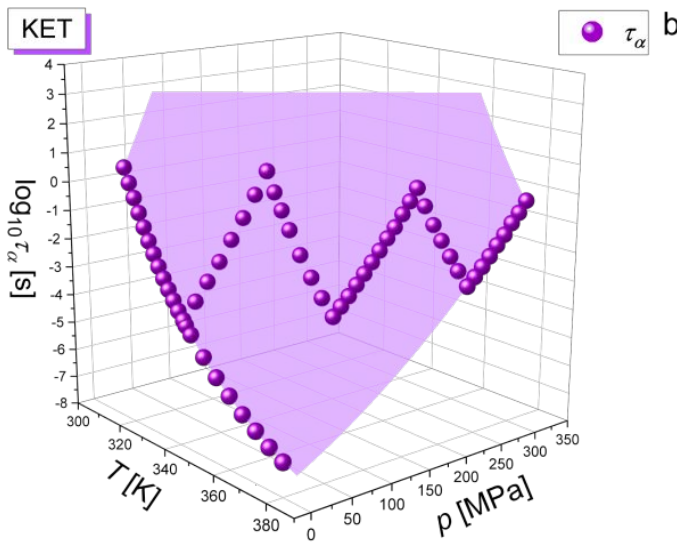

b)

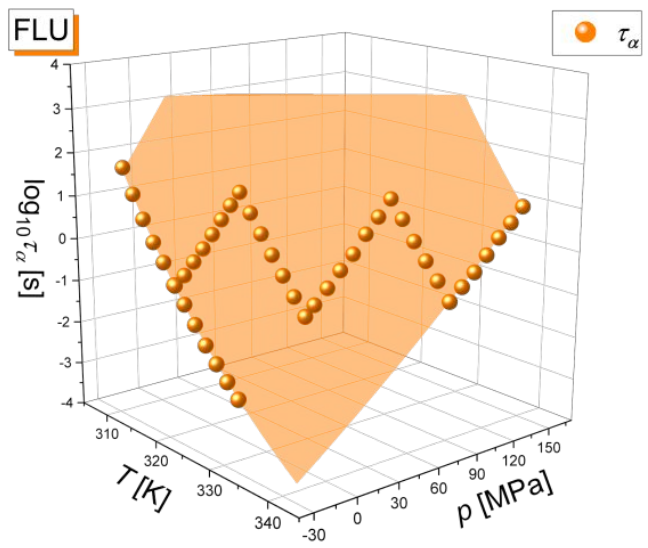

Figure S2. $\alpha$-relaxation times $\left(\log _{10} \tau_{\alpha}\right)$ plotted as a function of temperature $(T)$ and pressure $(p)$ for KET (panel a) and FLU (panel b). Coloured areas represent surface fits to Avramov equation (eq 2).

Table S1. Parameters of the modified Avramov equation (eq. 2) obtained from the analysis of $\tau_{\alpha}(T, p)$ dependencies for all APIs, and $\tau_{\delta / \alpha^{\prime}}(T, p)$ dependencies for ITZ, POS and TER.

\begin{tabular}{|c|c|c|c|c|c|}
\hline$\alpha$-relaxation & ITZ & POS & TER & KET & FLU \\
\hline $\log _{10}\left(\tau_{\infty}[\mathrm{s}]\right)$ & -7.14 & -7.50 & -9.08 & -9.11 & -9.32 \\
\hline$\alpha_{0}$ & 14.71 & 14.39 & 8.04 & 8.24 & 7.60 \\
\hline$C / C p_{0}$ & 0.318 & 0.214 & 0.146 & 0.189 & 0.171 \\
\hline$\Pi[\mathrm{MPa}]$ & 113.06 & 196.75 & 303.74 & 283.54 & 312.66 \\
\hline$\beta$ & 0.92 & 1.96 & 1.96 & 1.75 & 1.43 \\
\hline \multicolumn{5}{|l}{} \\
\hline$\delta / \alpha^{\prime}$-relaxation & ITZ & POS & TER & KET & FLU \\
\hline $\log _{10}\left(\tau_{\infty}[\mathrm{s}]\right)$ & -11.76 & -7.54 & -8.68 & --- & --- \\
\hline$\alpha_{0}$ & 4.29 & 11.32 & 6.71 & --- & --- \\
\hline$C / C p_{0}$ & 0.076 & 0.031 & 0.180 & --- & --- \\
\hline$\Pi[\mathrm{MPa}]$ & 235.19 & 315.69 & 241.46 & --- & --- \\
\hline$\beta$ & 0.87 & 2.67 & 1.32 & --- & --- \\
\hline
\end{tabular}



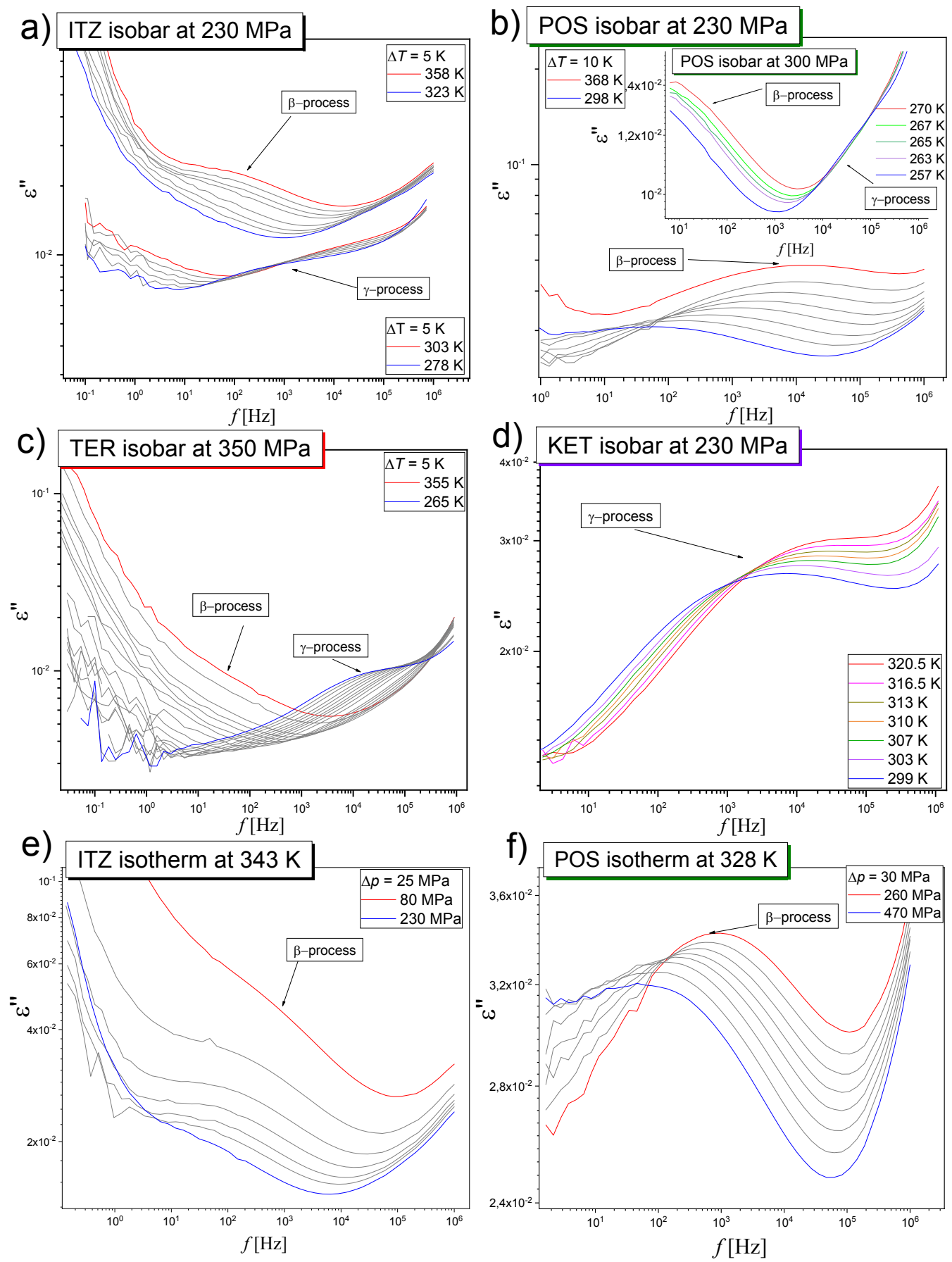

Figure S3. Representative dielectric loss spectra measured at constant pressure and indicated temperatures below the $T_{\mathrm{g}} / p_{\mathrm{g}}$ for ITZ (panel a), POS (panel b), TER (panel c), and KET (panel d). Panels $\mathrm{e}$ and $\mathrm{f}$ present isothermal data ( $T=$ const.) collected at indicated pressures for ITZ and POS, respectively. 